\title{
Smart Road Accident Detection using IoT Application
}

\author{
Shubham Mathur \\ B.E. Scholar, Department of Computer \\ Science \& Engineering, Bhopal, India \\ shubham.mathur196@gmail.com
}

\author{
Karishma Verma \\ B.E. Scholar, Department of Computer \\ Science \& Engineering, Bhopal, India \\ karishma18oct@gmail.com
}

\author{
Aayushi Priya \\ Department of Computer Science \& \\ Engineering, Bhopal, India \\ aayu.rec@gmail.com
}

\begin{abstract}
Road accidents rates are very high nowadays, especially two wheelers. Timely medical aid can help in saving lives. This system aims to alert the nearby medical center about the accident to provide immediate medical aid. The proposed framework automatically learns feature representation from the spatiotemporal features of pixel intensity. We consider the accident of the vehicles as an unusual incident. The possibility of an accident is determined based on the artificial intelligence approach and alarm is generated with preventive measures.
\end{abstract}

Keywords- Road Accident, Spatial, Temporal, Artificial Intelligence, IOT

\section{INTRODUCTION}

Smart cities are using various innovative technologies to improve the peoples quality of life [1], [2]. European Commission in 2010 [3] has launched one such highly visible and important initiative named European Initiative on Smart Cities. In smart cities sustainable transportation is a critical dimension where the goal is to build:

i. Intelligent public transportation systems based on realtime information,

ii. traffic management systems for congestion avoidance, and

iii. safety and green applications

However, the growing size of cities and increasing population mobility have determined a rapid increase in the number of vehicles on the roads, which has resulted in many challenges for road traffic management authorities among them road accidents require immediate attention to reduce the loss of life and properties. Traffic accidents caused an estimated 1.2 million deaths in 2004, with 50 million people injured [5]. Due to various security concerns, all the main cities across the world already installed a significant number of cameras for traffic monitoring purpose. The use of these already existing surveillance camera networks will be a viable solution, but these systems mostly rely on human observation. For human observers, it is almost impossible to monitor and recognize unusual events without missing in such a large number of camera scenes in real time [6]. Thus, it raises the need for automated solutions for accident detection.
Over the recent years, researchers from both industry and academia have been working to develop automatic detection methods using computer vision and pattern recognition techniques, but the level of current technology is still limited to apply them in the real world. Devising vision-based algorithm for this task is very challenging. In practice, the performance of computer vision based traffic accident detection algorithms can be challenged by many factors [7][9]. These factors include imaging conditions (varying illumination and changing weather conditions), environments (urban, highways), as shown in Fig. 1.

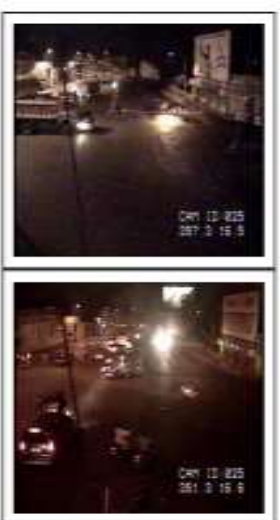

Night

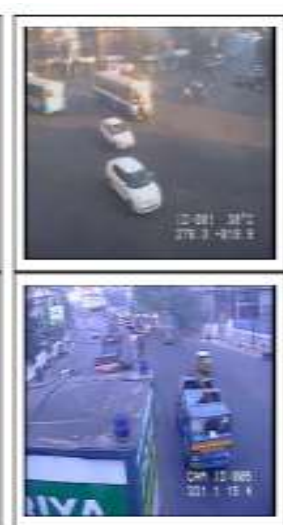

Early Morning

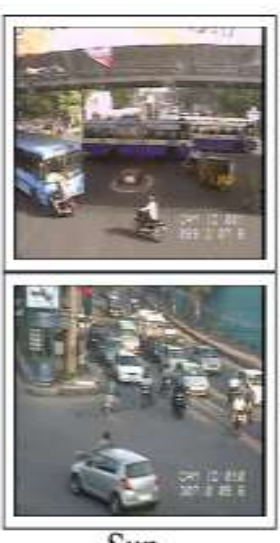

Sun
Figure 1: Video Samples under Different Environmental Conditions [1]

\section{II.CLASSIFICATION OF ROAD TRAFFIC ACCIDENTS}

A road traffic accident can be classified into following types: Pedestrians, pedal cyclists, motorcyclists, drivers of cars, commercial and passenger vehicles, animal vehicle collision, mass casualty incident, and Act of God [3], [4].

Pedestrians

Hitting a pedestrian while driving a car is very common. A study highlighted that Islamabad Police Department reported $53.3 \%$ fatalities for pedestrian cases that are $56 \%$ of reported fatal traffic accidents [5].

Pedal cyclists

Due to slow movement of pedal cyclists, they are not seen by vehicles which over takes another car and suddenly hit the 
pedal cyclists. As motor cycle moves much faster than cycle so motor cyclists are less vulnerable than pedal cyclists [6].

Motorcyclists

Riding a motorbike is a good and famous activity in most parts of the world due to less space requirements, high mileage and less polluting. If a vehicle hits a motorcyclist, due to the lack of safety features on a bike, rider will definitely get injured severely in most cases. The chance of as survival of motorcyclist is less compared to injured person in case a vehicle hit a car [7].

Drivers of cars, commercial and passenger vehicles

Most of the time, it is noticed that an accident occurs when a person is few miles away from his home [8]. The basic reasons of car accidents are over-speeding, casual behavior, reckless and unsafe driving. Table II shows common types of car accidents [9].

Animal Vehicle Collision

As the name implies it is a collision between an animal and a vehicle. Statistics shows that mortality of animals is increasing in North-East China due to accidents as compared to hunting [10]. Statistics shows that $67 \%$ drivers at Northern Tanzania felt that animal vehicle accidents were due to over speeding and night-time low visibility [11].

Mass Casualty Incident

It is an incident in which there are more casualties than a normal accident has [12]. These accidents include: Multiple vehicle collision, building collapse, mass transit accidents, HAZMAT (Hazardous Materials) incidents, WMD (weapon of mass destruction), multiple-shooting victims, and chemical exposure.

\section{III.ACCIDENT DETECTION STAGES}

The course of accident can be divided into three stages: precollision, collision, and post collision. Each stage gives us a significant amount of information but also involves several difficulties as discussed below.

\section{A. Pre-Collision}

The pre-collision case is the most vital information to explain an accident scenario. Also, this information may become a good evidence for crime scene investigation. The precollision situation is a clear violation of traffic rules by any/both the vehicles, which include violation of traffic lane, violation of signals at intersections, violation of speed limit at congested roads, abrupt motion on the road, etc. Finally, we can say that pre-collision stage is an unusual activity and thus can be easily detected by applying anomaly detection methods based on the various parameters, such as speed, trajectories, position, etc.

\section{B. Collision}

The collisions are essential to accident detection, but it is very complicated to detect and cannot be directly detectable by any general purpose computer vision technique. One way to detect a collision is to identify the joints of the trajectories of the vehicles over spatiotemporal dimensions. However, the major challenge is the discrimination between collision and occlusion. For this we use the trajectories over space-time interest points and improved dense trajectories.

\section{Post-collision}

As stated above that the collision and occlusions are hard to classify and may lead to false alarms. These false alarms further can be refined by considering the post collision scene. The two most common post-collision scenes include:

i. Fallen objects at the collision point: As we stated that the intersection of the trajectories of two vehicles might be a collision or an occlusion. However, after the intersection, if both the trajectories are continued, and no abrupt or zig-zag motion resulted. Then, the intersection is merely an occlusion, not a collision. However, if some abrupt motion or discontinued trajectories have occurred, then the possibility of a collision is high. Measure the time for which the object remains static.

ii. Crowd attention towards the collision point: The last and final stage of the accident is the crowded road or pedestrians running towards the collision point.

\section{IV.APPROACHES FOR ACCIDENT DETECTION}

The existing methods for traffic accident detection developed till date can be categorized into three approaches:

\section{A. Modeling of traffic flow patterns}

In this category, the typical law-full traffic patterns (namely, go-straight, U-turn, right-turn, etc.) are modeled as baseline and any deviation from this model is considered as an abnormal traffic event. This approach will work only when the normal traffic pattern appears at a fixed region repeatedly, thus unable to detect collisions which are essential to accident detection.

\section{B. Analysis of vehicle activities}

The methods in this categories first detect the moving vehicles and then extract motion features such as the distance between two vehicles, acceleration, direction, etc. of a vehicle from moving vehicles' tracks. However, unsatisfactory tracking performance in crowded traffic scenes becomes their bottleneck and limits their usage.

\section{Modeling of vehicle interactions}

These methods have been inspired by sociological concepts and model the interaction among vehicles and detect accidents. However, a large number of training data and use of speed change information alone limit the performance of these methods. 


\section{V.RELATED WORK}

The motor vehicle population is growing at a faster rate than the economic and population growth. Accidents and the death rate due to road accidents, especially two wheelers are also increasing at an alarming rate. Most of the accident deaths that happens are due to the lack of immediate medical assistance, on the roads like express highways. A facility for providing immediate medical assistance to the accident area can reduce the fatality to a greater extend. Thus comes the idea of an alert system that senses the accident and its seriousness to alert the nearby medical center for providing ambulance or medical aid to the accident area. The proposed system will check whether an accident has occurred and identify the seriousness of the injury to the accident victim/driver.

Once the decision of serious accident has taken, the system will check for the nearest medical center and notify them about the incident. The rescue team can rush to the spot immediately without any delay as the correct location will be communicated by the mobile phone of the accident victim. The system will also send message to the friends and relatives to inform them about the incident. Accident detection and alert system has been extensively studied over the past several years. Research work in this field has proposed a Telematics model which has three main modules [1].

The system is intended to capture the location of the vehicle through GPS receiver, send the location information to vehicle owner's mobile number through SMS and also to the telematics operator server through GPRS. Another prototype proposes a system to detect and provide faster assistance to traffic accident victims [2]. A prototype architecture to improve the chances of survival for passengers involved in car accidents has also been proposed [3].

Dinesh Singh et al [1] proposed a novel framework for automatic detection of road accidents in surveillance videos. The proposed framework automatically learns feature representation from the spatiotemporal volumes of raw pixel intensity instead of traditional hand-crafted features. We consider the accident of the vehicles as an unusual incident. The proposed framework extracts deep representation using denoising autoencoders trained over the normal traffic videos. The possibility of an accident is determined based on the reconstruction error and the likelihood of the deep representation. For the likelihood of the deep representation, an unsupervised model is trained using one class support vector machine. Also, the intersection points of the vehicle's trajectories are used to reduce the false alarm rate and increase the reliability of the overall system. We evaluated out proposed approach on real accident videos collected from the CCTV surveillance network of Hyderabad City in India. The experiments on these real accident videos demonstrate the efficacy of the proposed approach.
$\mathrm{Ki}$ and Lee [2] detects accidents by setting a predefined threshold on the certain parameters such as position, acceleration, and direction of vehicles. Moving objects are obtained by taking the difference between two successive frames.

A similar approach also presented by Hui et al. [3] where the parameters are computed from the trajectories of the moving vehicles obtained through background modeling using Gaussian mixture model (GMM) and tracking using mean shift algorithm. This method is simple and easy to implement and deploy but not suitable in unconstrained environments like a frequent change in traffic pattern and weather conditions since it relays only on the change in position and speed parameters. Also, the dependence only on the change in speed and position can easily lead to false alarms like a sudden movement of a vehicle.

Aköz and Karsligil [4] obtained moving vehicles using moving blob detection and tracking using Kanade-LucasTomasi (KLT) Tracker. The trajectories of various vehicles for normal traffic are clustered using continuous hidden Markov model (C-HMM) to set up a baseline of normal traffic which requires a large amount of training data to acquire all possible activity paths. If a given unseen trajectory shows significant deviation from baseline trajectories, then an accident is declared. Although, this method does not rely on speed parameters but the whole trajectory of a vehicle.

Sadek et al. [5] use histogram of flow gradient to obtain the orientation of flows. From the optical flow or velocity obtained, the Euclidean distances between the centers of gravity of patterns are calculated, and then logistic regression is used to predict the probability of the occurrence of an accident. But, this method uses logistic regression, the interpretation of which is merely a probability. The tracking of vehicles is a key component in the accident detection but tracking in the dense traffic and abrupt motion is a challenging problem because the scenario typically contains abrupt changes in the appearance and motion of the target.

A significant research is carried out in tracking under abrupt motion. Kwon and Lee [6] proposed a robust tracking method by alleviating the motion smoothness constraint in abrupt motion using Wang-Landau Monte Carlo (WLMC) sampling method in tracking algorithm.

Lim et al. [7] handle the tracking under abrupt motion by applying optimized swarm based sampling strategy for proposal selection.

$\mathrm{Su}$ et al. [8] used visual saliency model integrated with a particle filter for recovering lost track due to abrupt motion by detecting the target region from salient regions obtained from the saliency map of current frame.

These existing methods make use of motion or track of moving objects and simply try to define a normal baseline (many time using pre-decided threshold only) and any 
unknown event not obeying this baseline is simply declared as an accident. Although, the deviations in motion parameters gives useful pre-collision information but do not sufficient for accident detection.

Francesco Biral et al., [9] provided a novel intersection support (IS) system for motorcycles developed through the SAFERIDER project. The initial value of the longitudinal jerk (control input) of each plan is used as a measure of the correction that the rider has to apply to conform to an optimal-safe maneuver. Warning feedback is given to the rider by an appropriate combination of human- machine interface elements, such as the haptic throttle, the vibrating glove, and the visual display.

Ferhat Attal et al., [10] developed a methodology for riding patterns recognition based on a machine learning framework. The class of the riding situation is identified by using data collected from three-accelerometer and three gyroscope sensors mounted on the motorcycle.

Ferhat Attal et al., [11] presented a methodology that uses both acceleration and angular velocity signals to detect a fall of Powered Two Wheelers (PTW). The fall detection is therefore formulated as a sequential anomaly detection problem. The Multivariate Cumulative SUM (MCUSUM) control charts is used to detect such anomalies.

A framework was proposed by Amit Meena et al., [12], an Accident Detection Unit containing a GPS and GSM modem was used to sense and generate accidental events to a centralized server. The accidental situation is calculated by the acceleration and ground clearance of the vehicle. The GPS coordinates and time is sent to the accident detection server which maintains the historical data, current data and the rules that you configure in the system.

C. K. Harnett et al., [13] proposed a method with low-cost hardware for a generic WSN-to- Bluetooth gateway and open-source software that allows a wide subset of mobile phones to download and save WSN data.

Emmanouil N. Barmpounakis et al., [14], reviewed research conducted, thus far, on PTW and PTW oriented ITS services and applications, as well as to reveal possible directions on how PTW research could be supported by new technologies and improve macroscopic traffic conditions.

A system was proposed by Prachi R. Rajapollu et al., [15], forward a design for side stand automation by connecting a motor to the processor. The system along with the battery is kept inside a box. The box is provided with a lockable facility so that nobody else except the user can access it.

A design for accident detection and alert system in motorcycles was proposed by Fahim Bin Basheer et al., [16]. Three parameters were considered which include acceleration/ deceleration, tilt of the vehicle and the pressure change on the body of the vehicle.
In a system proposed by Manjunatha D et al.,[17], CAN protocol was implemented for monitoring of vehicle and sensor. Control Area Network is a serial bus standard for automotive application. The system includes accelerometer monitoring, engine temperature level and security for vehicle in parking areas. Shabnam Abtahi and his team from Distributed Collaborative Virtual Environment Research Laboratory University of Ottawa, Canada discussed a method for detecting drivers' drowsiness and subsequently alerting them. Their idea is to reduce the number of accidents due to drivers fatigue and hence increase the transportation safety. They proposed a method of yawning detection based on the changes in the mouth geometric features. Alcohol content in the driver's body is detected by means of an infrared breath analyzer placed at the steering wheel. The higher the concentration of ethanol, the more infrared absorption occurs [18].

Mugila. G et al.,[19], introduces a smart helmet system which detects that, the person wearing helmet or not and also the system detect the person is drunk, If the driver using cell phone during driving means the bike will be jammed slowly. In this system a transmitter is placed in the helmet and receiver at the bike. A switch will be there to ensure that the person is wearing the helmet or not. And also alcohol sensor is placed in the helmet near the mouth of the driver to check whether the driver is drunk.

S. Chandran et al., [20] introduced a smart helmet to provide a means and apparatus for detecting and reporting accidents. Sensors, Wi-Fi enabled processor and cloud computing infrastructures are utilized for building the system. The accident detection system communicates the accelerometer values to the processor which continuously monitors for variations. When an accident occurs, the related details are sent to the emergency contacts by utilizing a cloud-based service. The vehicle location is obtained by making use of the global positioning system.

Nagarjuna et al. [21] proposed an attempt to develop a car accident detection and communication system which will inform the relatives, nearest hospitals and police along with the location of the accident. The system sends the message to the stored emergency numbers successfully when the car is collided and toppled or tilted by more than 30 degrees.

\section{VI.IOT APPLICATION IN ACCIDENT DETECTION}

The Internet of Things (IoT) is the term used to refer to the communication between people to things and things to things. In today's society, technology is improving at an exponential rate. Broadband Internet is more widely available and more cost-efficient than ever before. Technology costs are going down and as of 2018, 36\% of the world's population use smartphones. The number of smartphone users worldwide is forecast to grow from 2.1 billion in 2016 to around 2.5 billion 
in 2019 [1]. IoT is the focus of research, and industries are investing heavily due to the potential benefits of IoT in various fields [2]. All of these things are creating a ripe environment for IoT. The health-care industry is benefiting from the technological advances that IoT has to offer with improved access to care, increased quality, efficiency, and reduced costs. As the technology for collecting, analyzing and transmitting data in the IoT continues to grow, more IoTdriven healthcare applications, services, and systems emerge [2]. Currently, many vehicles are equipped with an automatic crash response system that can communicate with a server in the Cloud alerting a paid provider of an emergency. Once the provider has been alerted, an operator communicates back with the driver to get further instruction and sends emergency personnel if necessary.

This research proposes a system that can eliminate the need for an operator. When the vehicle is in an accident it communicates directly with emergency services and family members giving the severity of the accident, GPS location, and the car ID. Ambulances are currently capable of sending patient information to the hospital. The uniqueness of this project is that sensors detect an accident and information is sent immediately to the ambulance, thus eliminating the need for an intermediary step.

\section{PROPOSED METHODOLOGY}

The existing system [1], an automatic response system is designed for post-collision accident detection and information for healthcare assistance. The use of sensors and microprocessor is done to detect an accident and send the location to the Cloud. From the Cloud, the notification is sent to the hospital, ambulance and emergency contacts. The system takes advantage of data such as position and location. The device is meant to immediately detect a collision involving the vehicle it is installed in. This is done using the accelerometer. The $\mathrm{x}$-axis refers to a forward or backward acceleration in order to detect front or rear end collision. The $y$-axis reading detects an impact from either side of the car. The $\mathrm{z}$-axis reading is used to detect a collision from above or below.

The proposed methodology is designed for detecting accident in pre-collision as well as post collision stage. The research work is focused on following:

i. The trajectory path of different vehicles are calculated and compared with other vehicles trajectories.

ii. If two trajectories collide then there is chance of accident. So, an alarm system will be generated for precollision stage.

iii. On occurrence of alarm, the preventive measures will be taken such as diverting the trajectory path, or reducing the vehicle speed.

In order to gain above objectives:
1. The proposed framework for automatic detection of accident incident composed of abnormality detection using the representation of spatiotemporal features of vehicles and collision detection using intersection points of trajectories.

2. The possibility of an accident is determined based on intersection of trajectories. This is performed using artificial intelligence approach.

3. Data is sent to cloud server with location of vehicle and alarm generation in pre-collision stage.

4. Similarly, in post collision stage, collision trajectories along with its GPS location is sent to cloud system.

\section{CONCLUSION}

Vision-based detection of road accidents using traffic surveillance video is a highly desirable but challenging task. Since this research work are using spatio-temporal features instead of entire frame or full video clip, it not only detects the accident but also able to localize the accident events. The method is able to detect accidents correctly with minimum false alarms on real accidents videos captured under various lighting conditions. However, challenges such as low visibility at night, occlusions, and large variations in the normal traffic pattern still pose significant challenges which are to be addressed.

\section{REFERENCES}

[1] Arif Shaik, Natalie Bowen, Jennifer Bole, Gary Kunzi, Danie Bruce, Ahmed Abdelgawad, Kumar Yelamarthi, "Smart Car: An IoT Based Accident Detection System", IEEE Global Conference on Internet of Things (GCIoT), 2018.

[2] Y. K. Ki and D. Y. Lee, "A traffic accident recording and reporting model at intersections," IEEE Trans. Intell. Transp. Syst., Vol. 8, No. 2, pp. 188-194, Jun. 2007.

[3] Z. Hui, X. Yaohua, M. Lu, and F. Jiansheng, "Vision-based realtime traffic accident detection," in Proc. 11th World Congr. Intell. Control Autom. (WCICA), Shenyang, China, Jun./Jul. 2014, pp. 1035-1038.

[4] Ö. Aköz and M. E. Karsligil, "Video-based traffic accident analysis at intersections using partial vehicle trajectories," in Proc. 17th IEEE Int. Conf. Image Process. (ICIP), Sep. 2010, pp. 4693 4696.

[5] S. Sadek, A. Al-hamadiy, B. Michaelisy, and U. Sayed, "Realtime automatic traffic accident recognition using HFG," in Proc. $20^{\text {th }}$ Int. Conf. Pattern Recognit. (ICPR), Istanbul, Turkey, Aug. 2010, pp. 3348-3351.

[6] J. Kwon and K. M. Lee, "Wang-Landau Monte Carlo-based tracking methods for abrupt motions," IEEE Trans. Pattern Anal. Mach. Intell., Vol. 35, No. 4, pp. 1011-1024, Apr. 2013.

[7] M. K. Lim, C. S. Chan, D. Monekosso, and P. Remagnino, "Refined particle swarm intelligence method for abrupt motion tracking," Inf. Sci., Vol. 283, pp. 267-287, Nov. 2014.

[8] Y. Su, Q. Zhao, L. Zhao, and D. Gu, "Abrupt motion tracking using a visual saliency embedded particle filter," Pattern Recognit., Vol. 47, No. 5, pp. 1826-1834, 2014.

[9] Francesco Biral, Roberto Lot, Stefano Rota, Marco Fontana, and VéroniqueHuth, "Intersection Support System for Powered Two Wheeled Vehicles: Threat Assessment Based on Receding Horizon Approach", IEEE Transactions On Intelligent Transportation Systems, Vol. 13, No. 2, pp 805-816, June, 2012.

[10] Ferhat Attal, Abderrahmane Boubezoul, Latifa Oukhellou, and Stephane Espie, "Riding patterns recognition for powered two wheelers users' behaviors analysis", Intelligent Transportation 
Systems (ITSC), 2013 16th International IEE Conference, pp 2033-2038, 2013.

[11] Ferhat Attal, Abderrahmane Boubezoul, Latifa Oukhellou, Nicolas Cheifetz and StéphaneEspié, "The Powered Two Wheelers fall detection using Multivariate Cumulative SUM (MCUSUM) control charts," Intelligent Transportation Systems (ITSC), 2014, 17th International IEEE Conference, pp 1280-1285, IEEE, 2014.

[12] AmitMeena, Srikrishnalyer, Monika Nimje, Saket JogJekar, Sachin Jagtap, Mujeeb Rahman, "Automatic Accident Detection and Reporting Framework for Two Wheelers", in IEEE International Conference on Advanced Communication Control and Computing Technologies (ICACCCT), pp 962-967, 2014.

[13] K. Harnett, "Open Wireless Sensor Network Telemetry Platform for Mobile Phones" IEEE Sensors Journal, Vol. 10, No. 6, pp. 1082- 1084, June 2010.

[14] Emmanouil N. Barmpounakis, Eleni I. Vlahogianni, and John C. Golias, "Intelligent Transportation Systems and Powered Two Wheelers Traffic" IEEE Transactions On Intelligent Transportation Systems,2015.

[15] Prachi R. Rajarapollu, Nutan V. Bansode, Pranoti P. Mane, "A Novel Two Wheeler Security System Based on Embedded System" 978-1- 5090-3480-2/16/ () IEEE.

[16] Fahim Bin Basheer, Jinu J Alias, Mohammed Favas C, Navas V, Naveed K Farhan, Raghu C V, "Design of Accident Detection and Alert System for Motor Cycles, pp 85-89, IEEE,2013.

[17] Manjunatha D, IshwarMalapur, Ganesh L Bhat, "Safety and Security for Two Wheeler Vehicle Using ARM Controller \& CAN protocol" International Research Journal of Engineering and Technology(IRJET) Volume: 03 Issue: 06, pp. 1082-1084, June, 2016.

[18] S. Abtahi, B. Hariri and S. Shirmohammadi, "Driver drowsiness monitoring based on yawning detection," 2011 IEEE International Instrumentation and Measurement Technology Conference, Binjiang, 2011, pp. 1-4.

[19] Mugila.G Muthulakshmi.M Santhiya.K, Prof.Dhivya.P "Smart Helmet System Using Alcohol Detection For Vehicle Protection," International Journal of Innovative Research in Science Engineering and Technology (IJIRTSE) ISSN: 2395-5619, Volume 2, Issue 7 July 2016.

[20] S. Chandran, S. Chandrasekar and N. E. Elizabeth, "Konnect: An Internet of Things(IoT) based smart helmet for accident detection and notification," 2016 IEEE Annual India Conference (INDICON), Bangalore, 2016, pp. 1-4.

[21] Nagarjuna R Vatti, PrasannaLakshmi Vatti, Rambabu Vatti, Chandrashekhar Garde, "Smart Road Accident Detection and communication System", International Conference on Current Trends towards Converging Technologies (ICCTCT), IEEE, 2018. 